\title{
COLON
}

\section{Absorption and metabolism of octanoate by the rat colon in vivo: concentration dependency and influence of alternative fuels}

\author{
J R Jørgensen, M D Fitch, P B Mortensen, S E Fleming
}

See end of article for authors' affiliations

....................

Correspondence to: S E Fleming, Department of Nutritional Sciences and Toxicology, University of California, Berkeley, CA 94720-3104, USA;

fleming@nature.berkeley.edu

Accepted for publication 18 October 2001
Gut $2002 \cdot 51 \cdot 76-81$

Background: Compared with short and long chain fatty acids, medium chain fatty acids (MCFAs) have been shown to provide the highest colonic absorption of substrate carbon. Moreover, colonic epithelial cells fulfil their basic energy requirements as easily from MCFAs as from short chain fatty acids. Aims: To further characterise octanoate as a colonic luminal substrate, we determined in vivo the influence of (i) substrate concentration and (ii) alternative luminal fuels, on rat colonic transport and metabolism

Methods: Segments of rat proximal colon $(8 \mathrm{~cm})$ were cannulated and perfused for 100 min with ${ }^{14} \mathrm{C}$ labelled octanoate. The right colic vein was also cannulated and venous blood analysed for total ${ }^{14} \mathrm{C}$, ${ }^{14} \mathrm{CO}_{2}$, and metabolites by scintillation counting and high performance liquid chromatography.

Results: Tracer appearance in mesenteric blood stabilised after 20-40 minutes of perfusion. Increasing luminal octanoate concentrations from 2 to $40 \mathrm{mM}$ resulted in linear increases in total carbon absorption. Maximum $\mathrm{CO}_{2}$ production was reached near $10 \mathrm{mM}$. A substantial proportion of octanoate was absorbed without being metabolised (59-94\%). The luminal presence of a mixture of alternative fuels had no influence on either octanoate transport or metabolism.

Conclusions: This study demonstrated substantial concentration dependent colonic absorption of octanoate, rendering this MCFA a potential and much needed high energy substrate for patients with compromised small bowel function. Moreover, octanoate meets the basic energy requirements of colonic epithelial cells in vivo as well as butyrate. This study also demonstrates the divergence of in vitro and in vivo data regarding fatty acid absorption and metabolism in the colonic epithelium.
S hort chain fatty acids (SCFAs), especially butyrate, are the main fuels of the colonic mucosa and play an important role in maintaining its structure and function. ${ }^{12}$ In addition, they are avidly absorbed by the colon of animals and humans. ${ }^{3}{ }^{4}$ Previous studies have indicated that the colon of animals and humans is also capable of absorbing medium chain fatty acids (MCFAs). ${ }^{5-10}$ By using our novel in vivo rat model, we have recently provided direct evidence that the colonic mucosa can both metabolise and transport MCFAs. ${ }^{11}$ Octanoate and decanoate were oxidised to $\mathrm{CO}_{2}$ just as well as butyrate and thus provided energy to the colonic epithelium. ${ }^{11}$ Maybe even more striking, the energy rich MCFAs provided 2-13-fold increases in total carbon transported into the bloodstream relative to equimolar concentrations of the SCFAs acetate and butyrate. ${ }^{11}$ Assuming that this carbon is eventually metabolised within the body, colonic absorption of MCFAs could provide approximately $20 \%$ of the basal metabolic needs of the animal. ${ }^{11}$ In patients with a shortened or malfunctioning small bowel, colonic absorption of dietary MCFAs may therefore significantly compensate for reduced absorptive capacity and contribute to their energetic needs. Hence the colonic capacity to absorb MCFAs, which is unknown, is of immediate clinical relevance and needs further investigation.

We have previously found that aspects of fatty acid metabolism are not always predicted from data using in vitro techniques, but require the use of an in vivo model. ${ }^{12}$ For example, the concentration needed to achieve maximum oxidation of butyrate in vivo was much higher than that reported for isolated colonic epithelial cells, ${ }^{12}$ and even exceeded concentrations seen in the colonic contents of rats, pigs, and monkeys ${ }^{13-17}$ In vivo data evaluating the ability of the colonic epithelium to metabolise MCFAs in vivo were however not fully evaluated.

In vivo studies on colonic transport and metabolism of fatty acids are few. ${ }^{11}{ }^{12}$ As administration of octanoate may be clinically useful to some patients by means of colonic absorption, we were therefore prompted to use our novel in vivo rat model to determine the influence of (i) substrate concentration and (ii) alternative luminal fuels on rat colonic transport and metabolism of octanoate.

\section{MATERIALS AND METHODS}

In this in vivo model, rats were surgically operated on to cannulate a segment of proximal colon, isolate the vasculature, and cannulate the right colic vein draining this segment. The lumen was then perfused with ${ }^{14} \mathrm{C}$ labelled octanoate solutions, and all blood draining the segment was collected from the right colic vein and analysed for total ${ }^{14} \mathrm{C}_{,}{ }^{14} \mathrm{CO}_{2}$, and metabolites. The experimental protocols are based on procedures that have been used previously. ${ }^{11}{ }^{12}$

\section{Chemicals}

$\left[1-{ }^{14} \mathrm{C}\right]$ labelled octanoate (sodium salt) was obtained from American Radiolabelled Chemicals, Inc. (St Louis, Missouri, USA). Unlabelled substrates (sodium salts), acetylcysteine, and antibiotics were obtained from Sigma Chemical Co., Inc.

Abbreviations: acyl-CoA, acyl-Coenzyme A; HPLC, high pressure liquid chromatography; $\mathrm{K}_{\mathrm{m}}$ the Michaelis-Menten constant; MCFAs, medium chain fatty acids; LCT, long chain triacylglycerol; MCT, medium chain triacylglycerol; SCFAs, short chain fatty acids; $V_{\max }$ maximum reaction rate. 
(St Louis, Missouri, USA). Sodium pentobarbital was obtained from Abbott Laboratories, Inc. (North Chicago, Illinois, USA). Sodium heparin was obtained from Elkins-Sinn, Inc. (Cherry Hill, New Jersey, USA).

\section{Animals}

Male Sprague-Dawley rats, six months of age, weighing 496 (8) g (mean (SEM)), were obtained from Simonsen Laboratories, Inc., (Gilroy, California, USA). All procedures involving animals were reviewed and approved by the Animal Care and Use Committee, University of California, Berkeley, California, USA.

\section{Substrates and solutions}

All substrates were prepared in Krebs-Henseleit buffer without calcium and with antibiotics and acetylcysteine, as previously described. ${ }^{12}$ Unlabelled octanoate was dissolved in this solution to produce final concentrations of 2, 5, 10, or 40 $\mathrm{mM}$ octanoate. Ethanolic solutions of $\left[1-{ }^{14} \mathrm{C}\right]$ labelled octanoate were evaporated to near dryness and then added in trace amounts to the solutions to produce an average specific activity of $0.08(0.02) \mu \mathrm{Ci} / \mu \mathrm{mol}$. Octanoate at $10 \mathrm{mM}$ was also tested in a mixture of $10 \mathrm{mM}$ each of unlabelled acetate, propionate, butyrate, glucose, and glutamine at a specific activity of $0.04(0.00) \mu \mathrm{Ci} / \mu \mathrm{mol}$ octanoate.

\section{Surgical procedure and lumen perfusion}

One rat was anaesthetised and placed on an operating pad with a rectal temperature probe. A nose cone ventilator provided oxygen while the respired gases were exhausted through a liquid trap to retain carbon dioxide for later ${ }^{14} \mathrm{C}$ analysis. An abdominal midline incision was made and the caecum was exteriorised onto a $37^{\circ} \mathrm{C}$ platform. The middle colic artery and vein were tied off and then cut to allow repositioning of the middle region of the colon within the body cavity. The right colic vein was then exposed. A cannula for infusion of substrate solution was inserted into the lumen of the colon segment at the position of the middle colic vein and secured tightly in place. A second cannula for the exit of effluent was inserted into the lumen of the proximal colon at the caecocolonic junction and also secured tightly. The segment was then flushed to clear it of excess mucus and any remaining digesta. The cannula supplying donor blood was installed into the left saphenous vein and another cannula was inserted into the aorta to measure blood pressure. Donor blood infusion rate was adjusted to maintain a systolic pressure of 160-180 mm Hg. Finally, a cannula was inserted into the right colic vein for blood collection.

The lumen was perfused at $1.0 \mathrm{ml} / \mathrm{min}$ with labelled substrate solution. Blood from the right colic vein was quantitatively collected over 10 minute intervals throughout the 100 minute perfusion period. Whole blood was analysed immediately after collection for ${ }^{14} \mathrm{C}$ and ${ }^{14} \mathrm{CO}_{2} \cdot{ }^{12}$ Expired ${ }^{14} \mathrm{CO}_{2}$ caught in the liquid trap was also quantitated.

\section{Blood metabolite analysis}

Metabolites for high pressure liquid chromatography (HPLC) analysis were extracted from frozen whole blood aliquots using a modified Bligh and Dyer methanol:chloroform procedure. ${ }^{18}$ Equal volumes of blood taken at time points 70 , 80 , and 90 minutes were pooled and spiked with a solution of unlabelled standards. The procedure has previously been described. ${ }^{11}$ All scintillation counting was performed in a 1600TR liquid scintillation counter (Packard Instr., Co.).

\section{Calculations}

Calculations were based on the specific activities of octanoate in solutions and the appearance of radioactivity in mesenteric blood, as described previously. ${ }^{11}{ }^{12}$ Data are reported as nmol octanoate carbon per gram wet weight per minute, where wet weight refers to the fresh weight of the blotted colonic segment exposed to the substrate.

\section{Experimental design and statistical analyses}

Fifteen rats were randomly assigned to five different substrate groups so that a total of three rats were exposed to each substrate solution. Because of unequal variance among substrates, statistical differences among group means were determined on $\log$ transformed data. When comparing total absorption of substrates and $\mathrm{CO}_{2}$ production, group means were based on the marginal mean value of three consecutive 10 minute intervals $(70,80$, and 90 minute time points) under steady state conditions (see results section), and a two way ANOVA on repeated measures was used. When comparing data from HPLC analysis of pooled blood, one way ANOVA was used. Differences were considered to be statistically significant at $\mathrm{p}<0.05$. The Tukey-HSD procedure was used as a follow up test for multiple comparisons. The statistical procedures in SPSS $^{19}$ were used to perform statistical analyses.

\section{RESULTS}

\section{Validity of the perfusion technique}

The cannulated colonic segments had a mean length of 7.7 (SEM 0.3$) \mathrm{cm}$ and a mean wet weight of $1.06(0.05) \mathrm{g}$, and consisted of the proximal third of the entire colon length (excluding the caecum). Sufficient substrate was perfused through the cannulated segment to prevent substantial changes in substrate concentration of the luminal perfusate and thereby maintain a constant availability of substrate to the epithelium during the experiments. On average, $<3 \%$ of the substrate was transported into mesenteric blood during the 100 minute perfusion period (octanoate in $2 \mathrm{mM}, 3.2$ (0.6)\%; in $5 \mathrm{mM}, 2.5$ (0.8)\%; in $10 \mathrm{mM}, 3.5$ (0.1)\%; in $40 \mathrm{mM}$, $2.3(0.2) \%$; and in $10 \mathrm{mM}$ in a mix, $3.0(0.2) \%)$.

Breath $\mathrm{CO}_{2}$ was quantitatively collected throughout the experiments. Blood samples were also taken from the aorta at the end of the experiments. The sum of radioactivity in breath $\mathrm{CO}_{2}$ and whole body blood taken from all experiments was 2.6 $(0.4) \%$ and $0.3(0.1) \%$, respectively, of the radioactivity transported into the mesenteric blood. Furthermore, significant differences were not seen for any of these two measures between substrate solutions, indicating that the procedure effectively prevented transport of luminal perfusate into tissues other than the cannulated intestinal segment, regardless of substrate concentration. Thus by restricting metabolism by other tissues, this method enabled us to accurately assess the fate of luminal octanoate in the perfused colonic segment. Of the total radioactivity that was perfused in substrate solutions, 101.7 (1.2)\% was recovered.

\section{Pooling of blood collected for HPLC analysis during steady state absorption}

Total octanoate carbon absorption from each substrate solution appeared to stabilise and reach steady state conditions after 20-40 minutes of perfusion (fig 1). Metabolism of octanoate to $\mathrm{CO}_{2}$ required approximately 40 minutes of perfusion to stabilise (fig 2 ). Thus data from three consecutive 10 minute intervals, corresponding to the 60-90 minutes of perfusion, were chosen for statistical analysis. For these 10 minute intervals, no substrate by time interaction was seen $(\mathrm{p}=0.64)$, allowing statistical comparison among the corresponding mean values for the five substrates (two way ANOVA). Correspondingly, no time effect within substrate was noted $(\mathrm{p}=0.25)$, showing that steady state absorption of substrates occurred during the 60-90 minute perfusion period. This allowed equivolumetric pooling of blood from this period for later HPLC analysis of metabolites. Steady state conditions for the 60-90 minute perfusion period were also achieved for the rate of substrate oxidised to $\mathrm{CO}_{2}$ (time interaction not statistically significant, $\mathrm{p}=0.20$ ) (fig 2 ). In addition, no substrate 


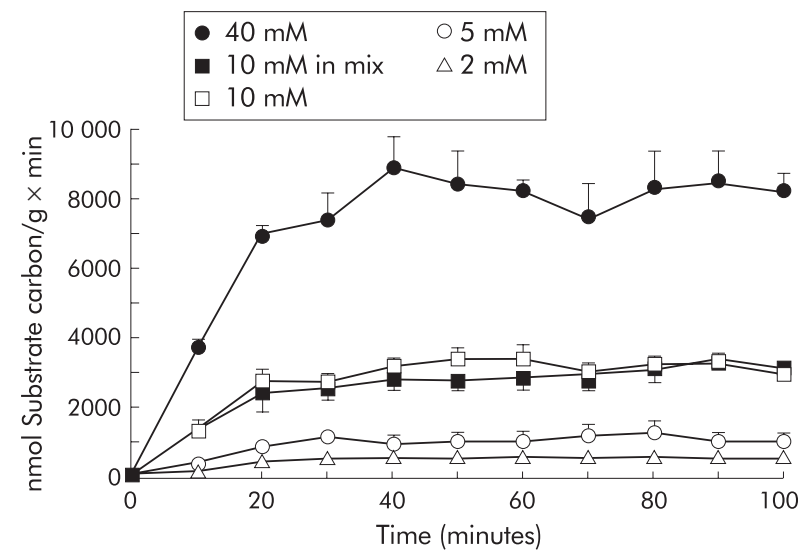

Figure 1 Influence of perfusion time on the rate of molar substrate carbon transport into mesenteric blood. Blood was collected at 10 minute intervals during the 100 minute perfusion period.

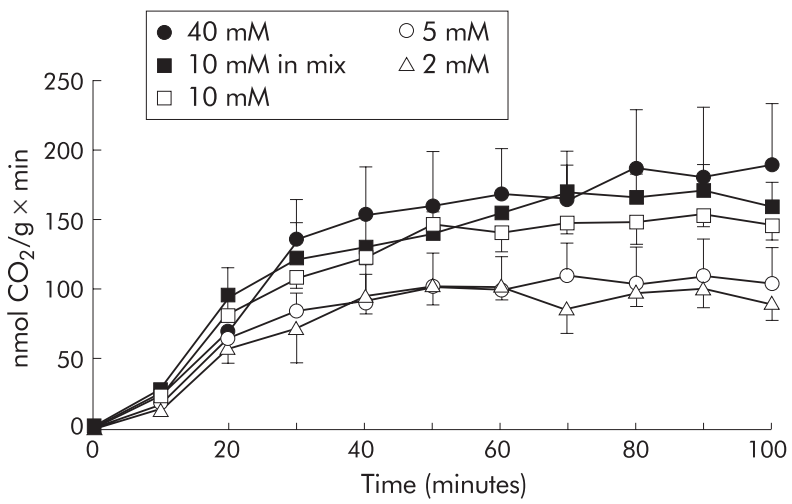

Figure 2 Influence of perfusion time on the rate of molar $\mathrm{CO}_{2}$ transport into mesenteric blood. Blood was collected at 10 minute intervals during the 100 minute perfusion period.

by time interaction was seen for this period $(p=0.50)$, allowing statistical analysis using a single mean steady state value.

\section{Influence of octanoate concentration on transport and metabolism}

Total octanoate carbon absorption increased linearly $(r=0.990$, $\mathrm{p}<0.01$ ) with increases in the octanoate concentration of the luminal perfusate (fig 3A). Also, the rate at which carbon was transported into mesenteric blood as octanoate increased linearly with luminal octanoate concentration $(r=0.991, \mathrm{p}<0.01)$ (fig $3 \mathrm{~B}$ ). Increasing the luminal octanoate concentration did not significantly increase metabolism of octanoate to $\mathrm{CO}_{2}$ $(\mathrm{p}=0.14$; fig $3 \mathrm{C})$ or to ketone bodies $(\mathrm{p}=0.31$; fig $3 \mathrm{D})$

\section{Influence of alternative luminal fuels on the transport and metabolism of octanoate}

The rate at which total octanoate carbon was absorbed from a mix of $10 \mathrm{mM}$ each of octanoate, acetate, propionate, butyrate, glucose, and glutamine was not significantly different from the rate of octanoate carbon absorption from $10 \mathrm{mM}$ octanoate alone (fig 4A). Similarly, the presence of alternative fuels in the luminal perfusate did not significantly influence the appearance in mesenteric blood of octanoate carbon absorbed as octanoate, $\mathrm{CO}_{2}$, or ketone bodies (fig $4 \mathrm{~B}-\mathrm{D}$ ).

\section{Relative absorption of unmetabolised substrate and metabolites}

The relative proportion of substrate transported into mesenteric blood without being metabolised was substantial, at $59 \%$ for $2 \mathrm{mM}$ octanoate (table 1). Relative proportions increased significantly with increasing concentrations of up to $94 \%$ for $40 \mathrm{mM}$ octanoate. The corresponding proportion of substrate oxidised to $\mathrm{CO}_{2}$ was $18 \%$ for $2 \mathrm{mM}$ octanoate, and decreased significantly with increasing concentrations to $2 \%$ for $40 \mathrm{mM}$ octanoate. Similarly, ketone bodies comprised 13\% of total octanoate carbon when octanoate was perfused at 2 $\mathrm{mM}$, and this proportion decreased significantly with increasing concentrations to $1 \%$ at $40 \mathrm{mM}$ octanoate. Small proportions of lactate and other metabolites accounted for the remaining substrate found in mesenteric blood. The presence of five other fuels in the luminal perfusate did not significantly influence the relative proportions of metabolites of octanoate in mesenteric blood.

\section{DISCUSSION}

The data reported here clearly demonstrate significant absorption of octanoate by the colon. More importantly, this study describes the concentration dependency of both octanoate transport into the blood and its metabolism, primarily to $\mathrm{CO}_{2}$ and ketone bodies, by epithelial tissues. The Michalis-Menten constant for oxidation of octanoate to $\mathrm{CO}_{2}$ in vivo is more than 16-fold higher than similar measurements using isolated colonic epithelial cells and is comparable with the in vivo value for butyrate. Also, contrary to previous in vitro studies, octanoate absorption in vivo was shown not to be affected by the simultaneous presence of other energy providing substrates. From these observations, it is likely that colonic absorption of MCFAs may be responsible for a large part of the benefit seen with oral medium chain triacylglycerol (MCT) therapy to patients with compromised small bowel function. ${ }^{20}$

The ability of the colon to absorb octanoate was directly demonstrated by measuring octanoate appearance in the mesenteric venous blood, along with its metabolites, over a wide concentration range (fig 3A). This luminal substrate was available to the epithelial cells for oxidation to $\mathrm{CO}_{2}$ and ketone bodies, and for other metabolic processes within the cell (table 1). Even at the lowest concentration however the majority was transported unmetabolised into venous blood for distribution throughout the body (fig 3B, table 1 ).

The maximum total absorption of octanoate carbon when provided at $40 \mathrm{mM}$ was 2.5 -fold higher than that of equimolar butyrate, measured previously using identical methodology. ${ }^{12}$ Similarly, the unmetabolised octanoate carbon transported into blood was 2.8-fold higher at a luminal concentration of $40 \mathrm{mM}$ compared with that of butyrate measured under the same conditions. ${ }^{12}$ Thus the potential for octanoate to provide energy to the whole body in patients with compromised small bowel function can be exploited using dietary modifications. MCTs are in fact already used in a range of malabsorptive disorders, as substitution of medium chain for long chain triacylglycerols (LCT) has been shown to reduce fat excretion. ${ }^{20}$ Although this reduction has been explained by a more rapid absorption of MCT than of LCT in the small bowel, ${ }^{21} 22$ colonic absorption of MCFAs may have been a contributing factor. This view is supported by a study in short bowel patients where substitution of MCT for $50 \%$ of existing dietary LCT was done. ${ }^{10}$ This dietary change resulted in luminal output concentrations of between 36 and $40 \mathrm{mM}$ of octanoate and decanoate, respectively, in patients without a colon in continuity, whereas concentrations were much lower for patients with a colon in continuity ( 4 and $10 \mathrm{mM}$, respectively). Hence in some instances appreciable MCFAs may be available to the colon, resulting in a significant gain in fat and energy absorption. ${ }^{10}$ As colonic absorption of MCFAs is also manifold greater than that of LCFAs, ${ }^{11}$ the potential energetic gain to the patient achieved by replacing LCT with MCT becomes increasingly important with concurrent decreasing small bowel function.

Oxidation of octanoate to $\mathrm{CO}_{2}$ by colonic epithelial cells in vivo reached maximum velocity $\left(\mathrm{V}_{\max }\right)$ at a concentration of 

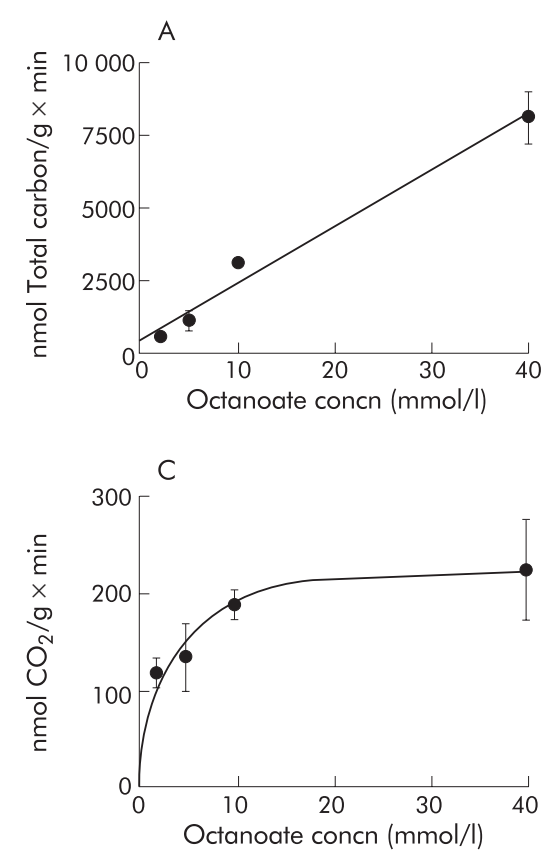

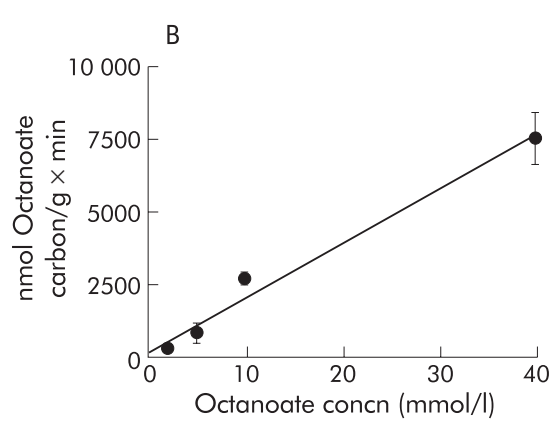

Figure 3 Influence of octanoate concentration on absorption and transport into mesenteric blood of total octanoate $(A)$, unmetabolised octanoate (B), carbon dioxide (C), and ketone bodies (D). approximately $10 \mathrm{mM}$. Although a precise value for the apparent Michalis-Menten constant $\left(\mathrm{K}_{\mathrm{m}}\right)$ cannot be calculated from the few data points, it is evident by extrapolation of the curve in fig 3C that $\mathrm{K}_{\mathrm{m}}$ is approximately $2.5 \mathrm{mM}$. These estimated $\mathrm{K}_{\mathrm{m}}$ and $\mathrm{V}_{\max }$ values are similar to those reported previously for butyrate oxidation by the colonic epithelium in vivo ${ }^{12}$ and demonstrate that octanoate can easily be used to meet the energy needs of epithelial cells. Thus a fundamental benefit of dietary MCT treatment may lie in the ability of the colon epithelium itself to utilise MCFAs as a primary substrate for maintenance of healthy tissue or for repair of damaged tissue.
Although the oxidation kinetics determined in this study agree well with previous values for butyrate in vivo, the $\mathrm{K}_{\mathrm{m}}$ differed dramatically from that determined using isolated colonic epithelial cells. ${ }^{23}$ In vitro, octanoate was found to be an excellent substrate with a $\mathrm{K}_{\mathrm{m}}$ equal to that of butyrate $(0.16$ $\mathrm{mM}$ and $0.13 \mathrm{mM}$, respectively, for octanoate and butyrate) and a maximum energy production from oxidation even exceeding that of butyrate. ${ }^{23}$ The current work showed that the concentration needed to achieve half maximum oxidation of octanoate was 16 -fold higher in vivo than when using isolated cells. This agrees with the result that the $\mathrm{K}_{\mathrm{m}}$ for butyrate
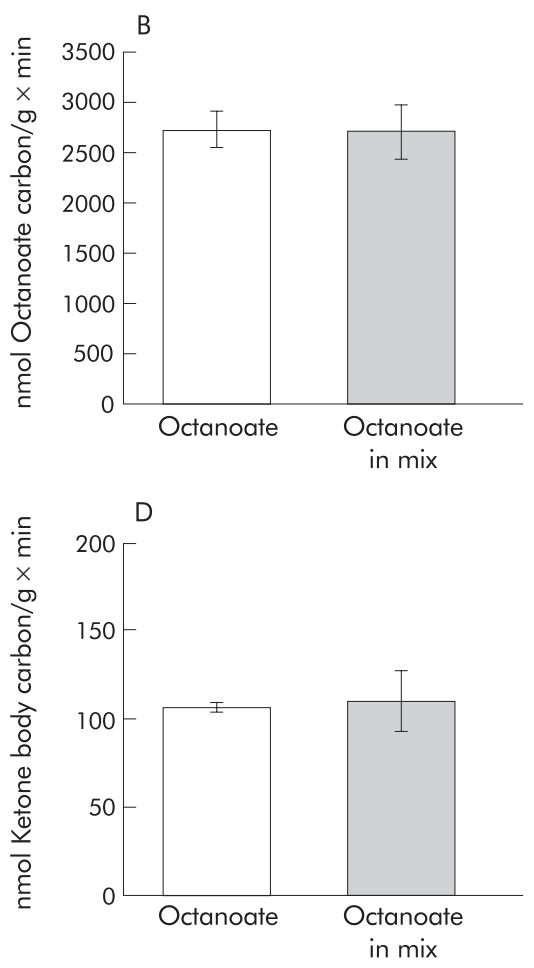

Figure 4 Influence of the simultaneous presence of other fuels on absorption and transport into mesenteric blood of total octanoate (A), unmetabolised octanoate (B), carbon dioxide (C), and ketone bodies (D). All substrates were present at $10 \mathrm{mM}$. The mix was composed of octanoate plus acetate, propionate, butyrate, glucose, and glutamine. 
Table 1 Relative proportions of octanoate carbon absorbed and transported into mesenteric blood during the 60-90 minute perfusion period of proximal rat colon

\begin{tabular}{|c|c|c|c|c|c|c|c|c|c|c|c|c|}
\hline \multirow{3}{*}{$\begin{array}{l}\begin{array}{l}\text { Concentration } \\
(\mathrm{mM})\end{array} \\
2\end{array}$} & \multirow{3}{*}{$\begin{array}{c}\text { Total } \\
100\end{array}$} & \multirow{2}{*}{\multicolumn{2}{|c|}{$\begin{array}{l}\text { Unmetabolised } \\
\text { substrate }\end{array}$}} & \multirow{2}{*}{\multicolumn{2}{|c|}{$\mathrm{CO}_{2}$}} & \multirow{2}{*}{\multicolumn{2}{|c|}{ Ketone bodies* }} & \multirow{2}{*}{\multicolumn{2}{|c|}{ Lactate }} & \multicolumn{3}{|c|}{ Other metabolites $†$} \\
\hline & & & & & & & & & & Known & \multicolumn{2}{|c|}{ Unknown } \\
\hline & & 58.9 & $(1.8)^{a}$ & 18.3 & $(1.1)^{d}$ & 13.3 & $(2.1)^{c}$ & 3.2 & $(0.3)^{c}$ & $2.1 \quad(0.2)^{c}$ & 4.2 & (0.2) \\
\hline 5 & 100 & 75.0 & $(2.7)^{b}$ & 10.1 & $(0.6)^{c}$ & 6.9 & $(1.4)^{\mathrm{b}}$ & 1.4 & $(0.1)^{\mathrm{b}}$ & $2.0(0.3)^{b c}$ & 4.6 & (1.2) \\
\hline 10 & 100 & 88.5 & $(0.8)^{c}$ & 5.0 & $(0.6)^{\mathrm{ab}}$ & 3.5 & $(0.1)^{\mathrm{ab}}$ & 0.6 & $(0.0)^{a}$ & $0.8(0.0)^{a}$ & 1.8 & $(0.1)$ \\
\hline 10 in mix $\ddagger$ & 100 & 86.5 & $(0.5)^{c}$ & 5.4 & $(0.4)^{\mathrm{b}}$ & 3.5 & $(0.2)^{\mathrm{ab}}$ & 0.6 & $(0.1)^{a}$ & $1.7(0.3)^{a b c}$ & 2.3 & (0.3) \\
\hline 40 & 100 & 93.6 & $(1.2)^{c}$ & 2.2 & $(0.3)^{\circ}$ & 0.8 & $(0.1)^{a}$ & 0.2 & $(0.1)^{a}$ & $1.1(0.2)^{\mathrm{ab}}$ & 2.1 & (0.7) \\
\hline $\mathrm{p}$ Value & - & \multicolumn{2}{|c|}{$<0.0001$} & \multicolumn{2}{|c|}{$<0.0001$} & \multicolumn{2}{|c|}{0.0001} & \multicolumn{2}{|c|}{$<0.0001$} & $<0.01$ & \multicolumn{2}{|c|}{$0.04 \S$} \\
\hline
\end{tabular}

Values are per cent (mean (SEM)); $\mathrm{n}=3$ for each substrate concentration. Values within columns with different superscripts are significantly different at $\mathrm{p}<0.05$.

${ }^{*}$ Acetoacetate $+3-\mathrm{OH}$ butyrate.

†The known fraction of other metabolites consists of acetate, propionate, butyrate, decanoate, and laurate. The unknown fractions were not further characterised.

tThe substrate "10 in mix" consists of $10 \mathrm{mM}$ of octanoate in a mixture of $10 \mathrm{mM}$ each of acetate, propionate, butyrate, glucose, and glutamine.

$\S$ Despite an overall $p$ value of 0.04 , none of the pairwise comparisons (Tukey) were significantly different.

oxidation was also manifold higher in vivo than in isolated colonocytes. ${ }^{12}$ The similarity in absorption and oxidation kinetics between octanoate and butyrate reinforces the finding that MCFAs can provide energy to the colon at levels comparable with or exceeding those of butyrate. The higher $\mathrm{K}_{\mathrm{m}}$ seen in vivo for both butyrate and octanoate may be due to a smaller surface area exposed to the substrate solution in the intact perfused colon. In vivo, only the epithelial surface is in contact with luminal contents, and this may be further influenced by a mucus layer. Absorbed substrate is easily transported out of the cell and into the bloodstream, keeping intracellular substrate concentrations below luminal concentrations. Isolated cells, exposed to substrate on all surfaces, are likely in near equilibrium with the surrounding substrate solution, and saturating conditions for oxidation can be reached at a lower solution concentration. Thus in vitro substrate absorption and oxidation rates measured using isolated cells do not always reflect in vivo conditions.

Another significant finding of the current work is that production of $\mathrm{CO}_{2}$ from oxidation of octanoate in vivo was not affected by the presence of other key nutrients. Absorption of total octanoate, transport of unmetabolised octanoate into the blood, and metabolism of octanoate to $\mathrm{CO}_{2}$ and ketone bodies were not affected when equimolar $(10 \mathrm{mM})$ amounts of acetate, propionate, butyrate, glucose, and glutamine were simultaneously present in the luminal perfusate (fig 4). This is in contrast with studies using isolated colonic epithelial cells which showed that butyrate inhibits the oxidation of octanoate and vice versa. ${ }^{23}$ The lower apparent $\mathrm{K}_{\mathrm{m}}$ for SCFAs and MCFAs in isolated cells allows saturating conditions to be achieved at much lower substrate concentrations and may accentuate the effects of competition. In vivo however concomitant influx of five other primary fuels resulted in no measured decreases in transport or metabolism, even when production of $\mathrm{CO}_{2}$ and ketone bodies from octanoate was at or near maximum. The ability to transport octanoate across the basolateral membrane into the blood may help keep substrate concentrations within the cell below saturation. Thus in vivo it appears that octanoate does not compete with SCFAs for activation to acyl-Coenzyme A (acyl-CoA). Neither does the acetyl-CoA derived from octanoate compete with other acetylCoA molecules for oxidation in the tricarboxylic acid cycle or for conversion to ketone bodies. This reinforces the idea that two distinct activating enzymes must be present in colonic epithelial cells: one which activates acetate and butyrate mitochondrially, and another which activates octanoate.

In summary, in vivo octanoate absorption in the proximal rat colon was concentration dependent. Octanoate was transported into venous blood predominantly unchanged while a portion was metabolised by the colonic epithelium, primarily to $\mathrm{CO}_{2}$ and to ketone bodies. Absorption, transport, and metabolism of octanoate were not affected by the simultaneous presence of other luminal substrates, including SCFAs, glucose, and glutamine. These results demonstrate that the colon can effectively use octanoate as oxidative fuel to meet its own energy demands and that it can absorb significant energy which can then be made available throughout the body. Colonic absorption of MCFAs is likely a major contributing factor to the success of oral MCT therapy in small bowel compromised patients.

\section{ACKNOWLEDGEMENTS}

The authors wish to thank Dr Mark Hudes, University of California, Berkeley, California, for statistical consultation. This study was supported by the following foundations: P Carl Petersen, Beckett, Codan, Ferring, Jacob Madsen and wife Olga Madsen, the Danish Foundation for the Advancement of Medical Science, and by the Danish Medical Research Council. Support was also received from the Agriculture Experiment Station (US).

\section{Authors' affiliations}

J R Jørgensen, P B Mortensen, Department of Medicine CA 2121 , Section of Gastroenterology, Copenhagen University Hospital, Rigshospital, Copenhagen, Denmark

M D Fitch, S E Fleming, Department of Nutritional Sciences and Toxicology, University of California, Berkeley, California, USA

\section{REFERENCES}

1 Fleming SE, Arce DS. Volatile fatty acids: their production, absorption, utilization, and roles in human health. Clin Gastroenterol 1986;15:787-814.

2 Fleming SE, Gill R. Aging stimulates fatty acid oxidation in rat colonocytes but does not influence the response to dietary fiber. $J$ Gerontol A Biol Sci Med Sci 1997;52:B318-30.

3 McNeil NI, Cummings JH, James WP. Short chain fatty acid absorption by the human large intestine. Gut 1978;19:819-22.

$4 \mathrm{McNeil}$ NI. The contribution of the large intestine to energy supplies in man. Am J Clin Nutr 1984;39:338-42.

5 Valdivieso VD, Schwabe AD. Absorption of medium-chain lipids from the rat cecum. Am J Dig Dis 1966;11:474-9.

6 Valdivieso V. Absorption of medium-chain triglycerides in animals with pancreatic atrophy. Am J Dig Dis 1972;17:129-37.

7 Pihl BG, Glotzer DJ, Patterson JF. Absorption of medium-chain fatty acids by the dog colon. J Appl Physiol 1966;21:1059-62.

8 Jørgensen J, Holtug K, Jeppesen PB, et al. Human rectal absorption of short- and medium-chain $\mathrm{C}_{2}-\mathrm{C}_{10}$ fatty acids. Scand J Gastroenterol 1998:33:590-4.

9 Linscheer WG, Castell DO, Platt RR. A new method for evaluation of portasystemic shunting. The rectal octanoate tolerance test. Gastroenterology 1969;57:415-23.

10 Jeppesen PB, Mortensen PB. The influence of a preserved colon on the absorption of medium chain fat in patients with small bowel resection. Gut 1998;43:478-83.

11 Jørgensen JR, Fitch MD, Mortensen PB, et al. In vivo absorption of medium-chain fatty acids by the rat colon exceeds that of short-chain fatty acids. Gastroenterology 2001;120:1152-61.

12 Fitch MD, Fleming SE. Metabolism of short-chain fatty acids by rat colonic mucosa in vivo. Am J Physiol 1999;277:G3 1-40 
13 Demigne C, Remesy C. Stimulation of absorption of volatile fatty acids and minerals in the cecum of rats adapted to a very high fiber diet. $J$ Nutr 1985; 115:53-60

14 Fleming SE, Fitch MD, Chansler MW. High-fiber diets: influence on characteristics of cecal digesta including short-chain fatty acid concentrations and $\mathrm{pH}$. Am J Clin Nutr 1989:50:93-9.

15 Holzgraefe DP, Fahey GC Jr, Jensen AH. Influence of dietary alfalfa: orchardgrass hay and lasalocid on in vitro estimates of dry matter digestibility and volatile fatty acid concentrations of cecal contents and rate of digesta passage in sows. J Anim Sci 1985;60:1235-46.

16 Costa MA, Mehta T, Males JR. Effects of dietary cellulose, psyllium husk and cholesterol level on fecal and colonic microbial metabolism in monkeys. J Nutr 1989;1 19:986-92.

17 Storer GB, Trimble RP, Illman RJ, et al. Effects of dietary oat bran and diabetes on plasma and caecal volatile fatty acids in the rat. Nutr Res 1983;3:519-26.
18 Kates M. Techniques of lipidology; isolation, analysis and identification of lipids. In: Work TS, Work E, eds. Laboratory techniques in biochemistry and molecular biology. New York: American Elsevie Publishing Co, 1975:351-2

19 SPSS. SPSSX users's guide, 2nd edn. Chicago, IL: SPSS, 1986

20 Ruppin DC, Middleton WRJ. Clinical use of medium chain triglycerides. Drugs 1980;20:216-24.

21 Greenberger NJ, Rodgers JB, Isselbacher KJ. Absorption of medium and long chain triacylglycerols: factors influencing their hydrolysis and transport. J Clin Invest 1966;45:217-27.

22 Clark SB, Holt PR. Rate-limiting steps in steady-state intestinal absorption of trioctanoin- $1-{ }^{14} \mathrm{C}$. Effect of biliary and pancreatic flow diversion. $J$ Clin Invest 1968;47:612-23

23 Jørgensen JR, Clausen MR, Mortensen PB. Oxidation of short- and medium-chain $\mathrm{C}_{2}$ - $\mathrm{C}_{8}$ fatty acids in Sprague-Dawley rat colonocytes. Gut 1997;40:400-5. 